\title{
Die Zukunft der Medizin in der Schweiz
}

H. Amstad, P. Suter

Im Sommer 1999 lancierte die Schweizerische Akademie der Medizinischen Wissenschaften (SAMW) das Projekt "Neu-Orientierung der Medizin». Ausgangspunkt war die Frage, ob die gegenwärtige Medizin in der Schweiz angesichts der vielfältigen gesellschaftlichen Wandlungen zeitgemäss und insbesondere zukunftsfähig sei. Am 30. August 2001 geht die SAMW mit dem Projekt an die Öffentlichkeit. Im Rahmen eines Symposiums in Bern präsentiert sie die Resultate und Schlussfolgerungen zweier Klausurtagungen und stellt sie zur Diskussion.

Die Diagnose des obersten Schweizer Arztes anlässlich einer ersten Klausurtagung des Projektes «NeuOrientierung der Medizin» war eindeutig. Aus Sicht von Dr. med. Hans Heinrich Brunner, Präsident der Verbindung der Schweizer Ärztinnen und Ärzte FMH, ist die ärztliche Tätigkeit zu einer Leistung mit Marktwert geworden. Nicht mehr Patientinnen und Patienten kämen zum Arzt, sondern Kundinnen und Kunden. Diese verlangten neben technischem Wissen zunehmend "existentielles Wissen" als Orientierungshilfe. Weil die traditionelle Medizin diesem Bedürfnis häufig nicht entsprechen könne, hinterlasse sie ein Vakuum, das von Heilsbringern aller Art gefüllt werde. Weitere, wesentliche Megatrends in der heutigen Medizin ortet Hans Heinrich Brunner in der Steuerung des menschlichen Organismus und seiner Reproduktion, in der Organtransplantation als Basis ewigen Lebens, in der Medikalisierung aller Lebensbereiche sowie in der Verknappung der Ressourcen bzw. der Ökonomisierung der Medizin.

Es waren ähnliche Überlegungen, die im Frühjahr 1999 Prof. Johannes Bircher veranlassten, dem Vorstand der SAMW die Schaffung eines "Think Tank" vorzuschlagen, der sich intensiv mit der Entwicklung der Medizin auseinandersetzen sollte. Nach intensiver Diskussion beschloss der Vorstand der SAMW im Sommer 1999 die Lancierung des Projektes «NeuOrientierung der Medizin» und beauftragte einen

Korrespondenz:

Dr. med. Hermann Amstad

SAMW

Petersplatz 13

CH-4051 Basel

E-mail: h.amstad@samw.ch

Steuerungsausschuss unter der Leitung von Prof. Bircher mit der weiteren Planung. Zwei Klausurtagungen im August 2000 und im Januar 2001 dienten dazu, in einem ersten Schritt die Situation der Medizin in der Schweiz zu analysieren, das heisst

- jene Bereiche zu identifizieren, in denen die Orientierung der gegenwärtigen Medizin als problematisch erlebt wird;

- die Mechanismen $\mathrm{zu}$ verstehen, welche $\mathrm{zu}$ Schwierigkeiten geführt haben;

- Wege zu skizzieren, auf denen eine Neu-Orientierung erreicht werden könnte.

\section{Die Resultate der Klausurtagungen}

Die Teilnehmenden an den beiden Klausurtagungen - rund 60 Persönlichkeiten aus Medizin, Gesundheitswesen, Pflege sowie medizinexternen Organisationen und Institutionen - waren sich einig, dass die Schweizer Medizin einen hohen Standard aufweist, dass aber in der Zielsetzung, Organisation und Umsetzung zahlreiche Verbesserungsmöglichkeiten existierten. Eine Analyse der Problemfelder der Medizin ergab sechs Kernbereiche, in denen Verbesserungen erwünscht sind. Im "Executive Summary» der beiden "Klausurtagungen» werden sie wie folgt aufgelistet:

Ziele der Medizin angesichts von Pluralität und Interessenkonflikten

Die gleichzeitige Orientierung der Medizin am Wohl jedes einzelnen Patienten / jeder einzelnen Patientin, an der sich wandelnden Wissenschaft, an den Bedürfnissen der Gesellschaft und an den ökonomischen Randbedingungen schafft Konflikte, die angegangen werden müssen. Die daraus resultierenden Spannungsfelder sind in einem für die Allgemeinheit transparenten Diskurs zu bearbeiten.

\section{Empowerment}

Stärker als bisher sollten Patientinnen und Patienten situationsgerecht und partnerschaftlich in Entscheidungsprozesse einbezogen werden. Der Zugang zu qualifizierten Informationen ("Wissen») über Gesundheit und Krankheit ist sicherzustellen. Gleichzeitig ist der Selbstverantwortung der Bürgerinnen und Bürger für ihre Gesundheit und bei der Behandlung von Krankheiten vermehrt Rechnung zu tragen.

\section{Entscheidungsgrundlagen}

In vielen Bereichen der Medizin sind die wissenschaftlichen Grundlagen unvollständig; ihre Erforschung ist dringend. Neue Erkenntnisse (z.B. Informatik, Genetik, Nanotechnik) sind mit kritischer Offenheit zu prüfen und zugänglich zu machen. Die Erarbeitung adäquater medizinischer Massnahmen darf sich aber nicht allein auf naturwissenschaftliche Argumente stützen, sondern muss auch sozial- und geisteswissenschaftliche Erkenntnisse berücksichtigen. 


\title{
Zukunft Medizin Schweiz
}

\author{
Das Projekt «Neu-Orientierung der Medizin» \\ Donnerstag, 30. August 2001, Kursaal, Bern
}

\author{
Programm \\ $09.30-09.45$ \\ $09.45-10.00$ \\ $10.00-10.20$ \\ $10.20-10.50$ \\ $10.50-11.20$ \\ $11.20-11.50$ \\ $11.50-12.30$ \\ $12.30-13.00$ \\ $13.00-14.15$ \\ $14.15-15.45$

\section{Begrüssung} \\ Neu-Orientierung der Medizin: ein Projekt der SAMW. Entstehung und Prozess \\ Neu-Orientierung der Medizin: was tut sich im internationalen Umfeld? \\ Was erwartet die Bevölkerung von der Medizin? \\ Ergebnisse einer repräsentativen Bevölkerungsbefragung in der Schweiz \\ Kaffeepause \\ Medizin und Gesundheitswesen: siamesische Zwillinge? \\ Ziele der Medizin - Grenzen der Medizin. \\ Resultate der Klausurtagungen und Perspektiven \\ Zum Verhältnis von Medizin und Gesundheit \\ Mittagessen \\ Wünschbar, realistisch, umsetzbar? \\ Resultate der Klausurtagungen auf dem Prüfstand dreier Podien
}

$15.45-16.15$

$16.15-17.15$

$17.15-17.30$

Kaffeepause

Die nächsten Schritte. Plenumsdiskussion

Konsequenzen für die SAMW
Prof. Werner Stauffacher, Basel

Prof. Johannes Bircher, Bern

Prof. Ambros Uchtenhagen, Zürich

Claude Longchamp, Bern;

Prof. Heidi Schelbert, Wasen

Dr. Ignazio Cassis, Bellinzona

Prof. Peter Suter, Genf

Bundesrätin Ruth Dreifuss, Bern

ModeratorInnen:

Prof. Iwan Rickenbacher, Schwyz; Ellinor von Kauffungen, Rheinau; Isabelle Moncada, Lausanne

Prof. Werner Stauffacher, Basel

Anmeldung: bis spätestens 15. August 2001 auf der Website der SAMW (www.samw.ch; «Zukunft Medizin CH»).

Es wird ein Unkostenbeitrag von Fr. 50.- (Mittagessen und Tagungsmappe) erhoben; Medizinstudentinnen/-studenten und Auszubildende gratis.

\section{Grenzen der Medizin}

Die Medizin stösst an biologische Grenzen (z.B. extreme Frühgeburten; hohes Alter), an die Grenze zwischen Machbarem und Sinnvollem (z.B. Intensivmedizin), an ethische Grenzen (z.B. Forschung) und an ökonomische Grenzen. Diese Grenzen zu akzeptieren ist gleichbedeutend damit, die Begrenztheit des Lebens zu akzeptieren. Entscheidungen, die angesichts dieser Grenzen getroffen werden müssen, sollten transparent und nachvollziehbar gestaltet werden.

\section{Anreize}

Das Gesundheitssystem beeinflusst die Medizin unter anderem durch das Schaffen von Anreizen. Diese sind so zu gestalten, dass qualitativ wertvolle und nachhaltige Leistungen in der Medizin sowie gesundheitsförderndes und präventives Verhalten des Bürgers und der Bürgerin belohnt werden.

\section{Lebenslanges Lernen}

Die Aus-, Weiter- und Fortbildung aller Medizinalpersonen sollte den neuen Erkenntnissen und den sich verändernden Anforderungen an die Medizinalberufe kontinuierlich angepasst werden. Besonderes Gewicht sollte der kommunikativen Kompetenz, der
Fähigkeit zum Umgang mit Konflikten, dem Genderaspekt, der Fähigkeit zum Umgang mit Fehlern, dem interdisziplinären Arbeiten und der Stärkung der Selbstverantwortung (Empowerment) beigemessen werden. Die bereits eingeleiteten Reformen des Medizinstudiums gehen in die richtige Richtung und verdienen Unterstützung.

Die oben skizzierten Kernbereiche dienten als Ausgangspunkt für einen Blick in die Zukunft und zur Erarbeitung eines Aktionsrahmens für die SAMW. Dabei kristallisierten sich vier Massnahmenbereiche heraus: Kommunikation mit dem gesellschaftlichen Umfeld; Initiieren einer Zieldiskussion innerhalb der Medizin; Erarbeitung neuer Richtlinien in dafür geeigneten Bereichen; Identifizierung und Anregung von Forschungsschwerpunkten.

\section{Die weiteren Schritte}

Am 30. August 2001 macht das Projekt mit dem Symposium "Zukunft Medizin Schweiz» den Schritt an die Öffentlichkeit (siehe Programm). Im Zentrum der Tagung stehen die Resultate und Schlussfolgerungen 
der Klausurtagungen sowie Vorschläge zur praktischen Umsetzung. Ebenfalls präsentiert werden die Ergebnisse einer grossen repräsentativen Bevölkerungsbefragung, mit der die Meinung des Durchschnittsschweizers / der Durchschnittsschweizerin zur Situation der Medizin erfasst wird. Der Einbettung des Projektes in den nationalen und internationalen Kontext gilt ein weiteres Referat, und Bundesrätin Ruth Dreifuss wird am Ende des Morgens zum Verhältnis von Medizin und Gesundheit sprechen. Der Nachmittag dient der vertiefenden Diskussion.

\section{L'avenir de la médecine en Suisse}

H. Amstad, P. Suter

En été 1999, l’Académie Suisse des Sciences Médicales (ASSM) lançait le projet intitulé «Nouvelle orientation de la médecine». Son point de départ était une question: compte tenu des multiples changements sociaux, la médecine en Suisse est-elle en accord avec son temps et, en particulier, est-elle bien armée pour l'avenir? Le 30 août 2001, l'ASSM rendra ce projet public: lors d'un symposium qui se tiendra à Berne, les résultats et conclusions de deux séminaires seront présentés et discutés.

Lors d'un premier séminaire de réflexion sur le projet "Nouvelle orientation de la médecine», le Dr Hans Heinrich Brunner, président de la Fédération des médecins suisses (FMH), a posé un diagnostic clair. De son point de vue, l'activité médicale est devenue une prestation à valeur marchande. Ce ne sont plus des patientes et patients qui vont chez le médecin, mais des clientes et clients, lesquels exigent de plus en plus, outre le savoir technique, un "savoir existentiel" pour les aider à s'orienter dans leur existence. Etant donné que la médecine traditionnelle n'est souvent pas en

Correspondance:

Dr Hermann Amstad

ASSM

Petersplatz 13

CH-4051 Basel

E-mail: h.amstad@samw.ch
Ziel des Symposiums im Kursaal Bern ist einerseits die Sensibilisierung einer breiten medizinischen Öffentlichkeit; diese soll die Möglichkeit erhalten, die bisherigen Resultate kennenzulernen und die daraus gezogenen Schlussfolgerungen zu bewerten. Andererseits soll die Basis gelegt werden für eine gemeinsame Plattform derjenigen Personen, Institutionen und Organisationen, denen die Neu-Orientierung der Medizin ein Anliegen ist. Die SAMW fühlt sich verpflichtet, ihre Erfahrung und ihre Ressourcen einzusetzen, um die Zukunft der Medizin mitzugestalten.

mesure de répondre à ce besoin, un vide s'est formé, que comblent des guérisseurs de toutes sortes. Pour Hans Heinrich Brunner, les autres grands axes de développement de la médecine actuelle sont le contrôle de l'organisme humain et de sa reproduction, la transplantation d'organes comme base d'une vie éternelle, la médicalisation de tous les domaines de la vie, ainsi que la raréfaction des ressources et l'«économisation" de la médecine.

Ce sont des considérations de ce genre qui ont amené le Professeur Johannes Bircher, au printemps 1999, à proposer au Comité de l'ASSM la mise sur pied d'une cellule de réflexion ("think tank") dont la tâche serait de se pencher intensément sur l'évolution de la médecine. Après une discussion approfondie, le Comité de l'ASSM a décidé en été 1999 le lancement du projet "Nouvelle orientation de la médecine" et chargé un comité de pilotage, placé sous la direction du Professeur Bircher, de poursuivre la préparation du projet. Dans une première phase, deux séminaires de réflexion organisés en août 2000 et en janvier 2001 ont permis d'analyser la situation de la médecine en Suisse, c'est-à-dire:

- d'identifier les domaines dans lesquels l'orientation actuelle de la médecine est ressentie comme problématique,

- de comprendre les mécanismes qui ont conduit à ces difficultés, et

- d'esquisser des pistes permettant d'engager la nouvelle orientation souhaitée.

\section{Les résultats des séminaires}

Les participantes et participants aux deux séminaires - une soixantaine de personnalités du monde médical, du système de santé, des soins infirmiers, ainsi que d'organisations et institutions externes à la médecine - ont été unanimes à considérer que la médecine en Suisse est d'un très haut niveau, mais aussi que de nombreuses possibilités existent pour l'amé- 
Symposium 2001 de l'Académie Suisse des Sciences Médicales

\title{
La médecine en Suisse demain
}

\author{
Le projet "Nouvelle orientation de la médecine» \\ Jeudi, 30 août 2001, Kursaal, Berne
}

\author{
Programme \\ $09.30-09.45$ \\ $09.45-10.00$ \\ $10.00-10.20$ \\ $10.20-10.50$ \\ $10.50-11.20$ \\ $11.20-11.50$ \\ $11.50-12.30$ \\ $12.30-13.00$ \\ $13.00-14.15$ \\ $14.15-15.45$ \\ Souhaits de bienvenue \\ Nouvelle orientation de la médecine: un projet de l'ASSM. Genèse et processus \\ Nouvelle orientation de la médecine: que se passe-t-il au niveau international? \\ Qu'attend le public de la médecine? \\ Résultats d'un sondage d'opinion représentatif mené en Suisse \\ Pause-café \\ Médecine et système de santé: des frères siamois? \\ Buts de la médecine - Limites de la médecine. \\ Résultats des séances de réflexion et perspectives \\ A propos de la relation entre médecine et santé \\ Repas \\ Souhaitable, réaliste, applicable? \\ Résultats des deux séminaires sous le regard critique de trois podiums
}

$15.45-16.15$

$16.15-17.15$

$17.15-17.30$

Pause-café

Les prochaines étapes. Discussion en plénum

Conséquences pour l'ASSM
Prof. Werner Stauffacher, Bâle

Prof. Johannes Bircher, Berne

Prof. Ambros Uchtenhagen, Zurich

Claude Longchamp, Berne;

Prof. Heidi Schelbert, Wasen

Dr Ignazio Cassis, Bellinzone

Prof. Peter Suter, Genève

Mme la conseillère fédérale

Ruth Dreifuss, Berne

Modérateurs:

Prof. Iwan Rickenbacher, Schwyz;

Ellinor von Kauffungen, Rheinau;

Isabelle Moncada, Lausanne

Prof. Werner Stauffacher, Bâle

Inscription: jusqu'au 15 août 2001 sur le site Internet de l'ASSM (www.assm.ch; «La médecine en Suisse demain»).

Une participation de Fr. 50.- (déjeuner et documentation compris) est demandée, sauf pour les personnes en formation.

liorer, que ce soit dans ses buts, son organisation ou sa mise en œuvre. L'analyse des problèmes de la médecine a débouché sur l'identification de six domaines clés dans lesquels des améliorations sont souhaitables. Dans le rapport consacré aux deux séminaires («Executive summary»), ces domaines clés sont exposés comme suit:

Buts de la médecine dans l'optique de la pluralité et des conflits d'intérêts

Du fait que la médecine est orientée à la fois sur le bien de chaque patiente et patient, sur une science en constante évolution, sur les besoins de la société et sur les conditions cadres économiques, des conflits surgissent, qui doivent être réglés. Les tensions qui en découlent doivent faire l'objet d'un débat transparent pour tout le monde.

\section{«Empowerment»}

En fonction de la situation, les patientes et patients doivent être davantage associés aux processus de décision en tant que partenaires. L'accès aux informations qualifiées ("savoir») sur la santé et la maladie doit être garanti. En même temps, la responsabilisation individuelle des citoyennes et citoyens à l'égard de leur santé et d'un éventuel traitement médical doit être favorisée.

\section{Bases de décision}

Dans de nombreux secteurs de la médecine, les bases scientifiques sont incomplètes; il est urgent d'intensifier la recherche dans ces secteurs. Les nouveaux savoirs (p. ex. informatique, génétique, nanotechnologie) doivent être examinés avec un esprit ouvert et critique, et rendus accessibles. L'élaboration de mesures médicales adéquates ne doit toutefois pas s'appuyer seulement sur des arguments relevant des sciences naturelles, mais tenir compte aussi des connaissances des sciences humaines et sociales.

\section{Limites de la médecine}

La médecine se heurte à des limites biologiques (p. ex. naissances de prématurés extrêmes; grand âge), à des limites entre ce qui est faisable et ce qui est souhaitable (p. ex. médecine intensive), à des limites éthiques (p. ex. recherche) et à des limites économiques. Accepter ces limites revient à accepter le caractère limité de la vie. Les décisions prises en fonction de ces limites doivent être transparentes et compréhensibles. 


\section{Incitations}

Le système de santé influence la médecine notamment à travers des incitations. Ces incitations doivent être organisées de manière à récompenser les prestations médicales orientées sur la qualité et la durabilité, de même que les comportements des citoyennes et citoyens reflétant un souci de préservation de la santé et de prévention des maladies et accidents.

\section{Apprentissage tout au long de la vie}

La formation, le perfectionnement et la formation continue des professionnels de la médecine doivent être adaptés en permanence à l'état des connaissances et aux exigences changeantes à l'égard des professions médicales. Une attention particulière doit être accordée à la compétence de communication, à la capacité de régler les conflits, aux aspects gendre, à la capacité de gérer les erreurs, aux travaux interdisciplinaires et au renforcement de la responsabilisation individuelle («empowerment»). Les réformes déjà engagées dans le domaine des études de médecine vont dans la bonne direction et méritent d'être soutenus.

Ces domaines clés ont servi de point de départ à la réflexion sur l'avenir et à l'élaboration d'un cadre d'action pour l'ASSM. Quatre domaines de mesures ont été définis: communication avec l'environnement social; lancement d'un débat au sein du monde médical sur les buts de la médecine; élaboration de nouvelles directives dans des domaines qui s'y prêtent; identification et proposition de points forts pour la recherche.

\section{Les prochaines étapes}

Le 30 août 2001, le projet sera porté sur la scène publique dans le cadre du symposium "La médecine en Suisse demain" (voir programme). Ce forum sera centré sur les résultats et conclusions des séminaires, ainsi que sur les propositions d'application pratique. Seront également présentés à cette occasion les résultats d'un grand sondage représentatif qui permettra de mieux cerner l'opinion de la population suisse sur la situation de la médecine. Un autre exposé sera consacré à l'intégration du projet dans le contexte national et international. En outre, vers la fin de la matinée, la Conseillère fédérale Ruth Dreifuss parlera de la relation entre médecine et santé. Au cours de l'après-midi, un débat approfondi aura lieu.

Le but de ce symposium est double: d'une part, sensibiliser un large public médical en lui donnant la possibilité de prendre connaissance des résultats obtenus jusqu'ici et d'évaluer les conclusions qui en ont été tirées; d'autre part, poser les bases d'une plate-forme commune réunissant toutes les personnes, institutions et organisations intéressées par la nouvelle orientation de la médecine. Compte tenu de la responsabilité dont elle est investie, l'ASSM ne saurait éviter de mettre à profit son expérience et ses ressources en vue de contribuer à l'avenir de la médecine. 